\title{
Forms of Collaboration in Collaborative Enterprises: An Ontological Model
}

\author{
Barbara Livieri $^{1}$, Marco Zappatore ${ }^{2}$, and Mario Bochicchio ${ }^{2}$ \\ ${ }^{1}$ Department of Economic Sciences \\ ${ }^{2}$ Department of Engineering \\ University of Salento, Lecce, Italy \\ \{barbara.livieri, marcozappatore, mario.bochicchio\}@unisalento.it
}

\begin{abstract}
Reasoning techniques and their relations to cognition and reality gained significant momentum fueled by the advent of complex information systems which rely on robust and coherent, formal representations of their subject matter. In this sense, ontologies can provide models of different aspects of a business entity contributing to intra- and inter-enterprise information systems. This is particularly relevant for collaborative enterprises, which are entities far more complex than single enterprises. In particular, for the governance of collaborative enterprises it is essential to determine the relevant KPIs for the type of collaborative enterprise, taking into account several characteristics such as lifecycle and size. The main objective of this work is to develop an ontology of collaborative enterprises which enables the semi-automatic classification of instances. To this aim, we take into account the type of collaborative enterprise, its maturity (i.e., the lifecycle phase), size, territorial extension and other characteristics.
\end{abstract}

Keywords: Collaborative Enterprises, Ontologies, Enterprise Modeling, Business Rule.

\section{Introduction}

The transition from intra-organizational to inter-organizational relationships characterized the last twenty years of the evolution of enterprises. More recently the increasing importance of Collaborative Enterprises (CEs) and the growing impact of technologies on businesses drove the transition towards a new phase of trans-organizational relationship, characterized by an increased speed to create value [1, 2]. Indeed, CEs, defined as "a number of autonomous organizations working together [...] for mutual benefit" [3], are often used as catalysts of competitive advantages.

However, it is known that globally $50 \%-70 \%$ of CEs fails, often due to the lack of a comprehensive analysis that combine strategic goals and KPIs [4, 5] with a possible negative impact on component firms. Indeed, CEs are heterogeneous clusters of partnerships among enterprises. CEs can be of different types (e.g., horizontal or vertical) and have different goals; therefore, they need for different KPIs [6]. In other words, firms and CEs have to understand which KPIs are relevant and what a KPI means in a 
given $\mathrm{CE}$ with defined set of goals. However, this kind of understanding is not immediate, especially in several SMEs, which lack of the know-how needed to perform this kind of analysis and often choose the more "known" KPI, instead of the more relevant, with possible negative effects on the CE equilibrium. In order to measure performance and to create governance tools for inter-organizational environments, it is essential to understand which KPIs are useful for which types of CE [7].

In this scenario, ontologies and reasoning techniques can help in the understanding of complex entities and in the development of specific Enterprise Information Systems for intra- and inter-organizational settings. By committing to the same ontological specification, different applications share a common vocabulary with a formal language and clear semantics. Also, by representing knowledge with a wellestablished formalism [8], internal consistency and compliance checking can be performed in order to determine content adequacy. The main objective of this work is to develop an ontology of CEs, starting from the taxonomy of Collaborative Networks (CNs) developed by [9], and to infer knowledge from a domain body. To this aim, we take into account the type of a CE, its maturity (i.e., the lifecycle phase), size and other characteristics. The work is structured as follows. In Section 2 we analyze related works on enterprise modeling. Section 3 is for the modeling methods and Section 4 for the representation of the ontology and the analysis of the case study. Finally, in Section 6 we discuss the ontology and draw conclusions.

\section{Related Works}

Ontologies can be very effective to represent shared conceptualizations of specific domains [10]. To this aim, ontologies can be useful, since they can be seen as repositories of concepts, intended as complex information structures tightly interconnected with each other, on which reasoning functionalities can be applied.

An ontology for CNs has been proposed in [11], where the organizational structure and the domain specific knowledge of Virtual Breeding Environments (VBEs) are represented. In particular, each VBE has some assets and have some participants. Each VBE participant has a VBE Role and to each role some tasks are associated. Also, a VBE has some business opportunities related to the development and commercialization of products and services. VBEs are defined as organizations, to which are connected competencies and processes. In turn, each Process uses some resources and produce or use as inputs other products and services. Although this ontology provides a general representation of CNOs and VBEs, it is possible to analyze the roles of participants but not to understand CEs types, since the ontology is focused on VBE. Therefore, the ontology is unable to provide a more comprehensive classification of CESs. The same considerations apply to the ontology for VBE capabilities and profiles [12]. Moreover, in [9], a taxonomy of Collaborative Networks (CNs) is provided. The authors start from a definition of CNs in order to classify 26 types of CNs, among which digital ecosystems. Although the study is quite interesting and takes into account criteria such as the time perspective, the analysis should be broadened. Also, the lack of a formal language doesn't allow the semi-automatic classification of 
instances (CEs). In [13] the IDEON ontology is proposed in order to support the design and the management of collaborative and distributed enterprises. To this aim, the authors take into account four views of the collaboration, namely, a) Enterprise Context View; b) Enterprise Organizational View; c) Process View; and d) Resource/Product View. Finally, in [14] a model for supply chain is presented with the aim of enabling the semantic integration of Information Systems. In order to do so, some basic concepts, such as the supply chain structure (SC_Structure), the participants (Party), their roles (Role), the purpose of the alliance (Purpose), the Activity, the Resource, the Performance and the Performance_Metric. However, not even in these two cases there is a classification of the types of CEs, although the basic concepts used in these ontology can be borrowed

\section{Motivation and Requirements}

This works is part of a wider project regarding performance measurement in CEs. This is a non-trivial task, which has not yet been addressed by current literature [15, 16]. In this context of high-complexity, enterprise modeling techniques can be helpful [17]. In more detail, in our proposal, the performance measurement is based on the definition of domain-specific KPIs with the aim of supporting the management of CEs. Domain-specific KPIs are derived starting from the analysis of the CEs and of the participants' goals and form the type of CE. Indeed, there are several kinds of CEs, which need different KPIs [3]. Therefore, it is particularly relevant to classify CEs in order to identify the peculiarities of each type and, consequently, to offer specific support for the measurement of performance. Using this scenario as a starting point, a set of competencies questions, subdivided in simple (SCQ) and complex (CCQ), were elicited. As an example, we provide the following:

[SCQ.1] which types of alliances are possible?

[SCQ.2] what characteristics should a CE satisfy in order to be a strategic horizontal alliance?

[SCQ.3] which organizational structures are possible?

[CCQ.1] how many firms should a CE be composed of, to have a dyadic structure?

[CCQ.2] what is the size of a collaborative enterprise with more than 10 firms?

[CCQ.3] what type of CE is the one that has a time perspective of more than 3 years and a vertical integration?

\section{Modeling Methods}

When dealing with ontologies, the first trade-off to be taken into account is between their level of semantic description and their level of decidability (i.e., the capability of specific software applications, the reasoners, to produce new knowledge by inferencing over the explicitly defined semantic relationships). Different ontology languages and flavors are available so that the modeler can tune its knowledge base properly. For such reasons we selected the OWL 2 DL language [18] since it is fully decidable. 
In addition, OWL DL can be combined with SWRL, a language for the Semantic Web used to express rules as well as logic.

Moreover, knowledge resources can be represented as classes or instances depending on specific modeling requirements. We adopted a class-based approach to model the CE macro types and their characteristics, while we used business rules based on SWRL in order to infer the CE macro-types from the association of an instance of the class $\mathrm{CE}$ with some characteristics. In this way, end-users can easily populate the ontology by adding instances to CE classes, which inherit the semantic structure associated to the CE class they belong to. The ontology library named "CE Ontology" was developed with a modular architecture, as modularity is advantageous in terms of portability and reuse, domain decomposition and content categorization [19]. We modeled a core ontology (ontoPM) and a set of domain-dependent modules, namely ontoKPI for Key Performance Indicators, ontoFirm and ontoCE, which gather concepts describing firms and CEs respectively. The adoption of core and domain ontologies is a well-established custom in ontological engineering [20, 21]. CE ontology was developed by using the Protégé Desktop editor (v.4.3) [22], the de-facto freeware open-source ontology modeling tool. Ontology consistency, class expression satisfiability and finite reasoning time complexity were checked by using Pellet reasoner 2.2.0 [23]. The DL expressivity of the ontology is SHROIQ(D).

\section{Collaborative Enterprise Ontology}

This section provides some ontology fragments exemplifying our modeling approach.

The first excerpt illustrates CollaborativeEnterprise, the class describing a $\mathrm{CE}$, its subclasses and associations with other classes. As depicted in Fig. 1, each CE has a CELifecyclePhase, an Organizationalstructure and a Size (small, medium, large). This is codified by using existential (owl:someValuesFrom) or universal property restrictions (owl:allValuesFrom). Also, each CE is composed by at least 2 firms, which is codified with owl:ObjectMinCardinality and it has Relation and Alliance as subclasses. In turn, Alliance has an object property based semantic characterization, involving integrationLevel (low, medium, high), timeHorizon (shortTerm, mediumTerm, longTerm) and integrationType (horizontalintergration or verticalIntergration. Finally, Alliance have as subclasses StrategicAlliance and TacticAlliance.

As show in Fig 2, the CE classification based on size, time horizon, type (strategic or tactic, horizontal or vertical) and organizational structure is performed with reasoning support [24], which enables to infer new knowledge from the original domain body. To do so, we used functional dependencies, represented by means of business rules, coded with Semantic Web Rule Language (SWRL) [25]. In particular, in order to determine the size of a $\mathrm{CE}$, we use the datatype property hasNumberOfFirms and we define the following rule: CollaborativeEnterprise (?x), hasNumberofFirms(?x, ?integer), greaterThan(?integer, 4), lessThanorEqual (?integer, 10) $->$ hasSize(?x, Medium). In this way, we assert that if a $\mathrm{CE}$ has a number of firms greater than 4 but less or equal to 10 , then it has a medium 
size. Similar rules have been used in order to define the small and the large size. The timeHorizon has been determined by means of the datatype property hasTimePerspective and of the following rule: Alliance (?x), hasTimePerspective(?x, ?integer), greaterThan(?integer, 3) -> hastimeHorizon (?x, longTerm). Through this rule we assert that if an alliance has a time perspective greater than 3 years, then it has a long term horizon.

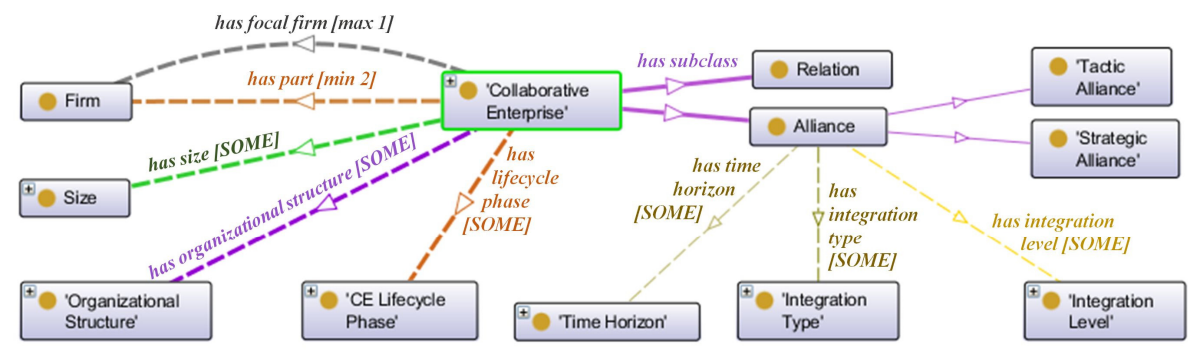

Fig. 1. Representation of a fragment of the ontology

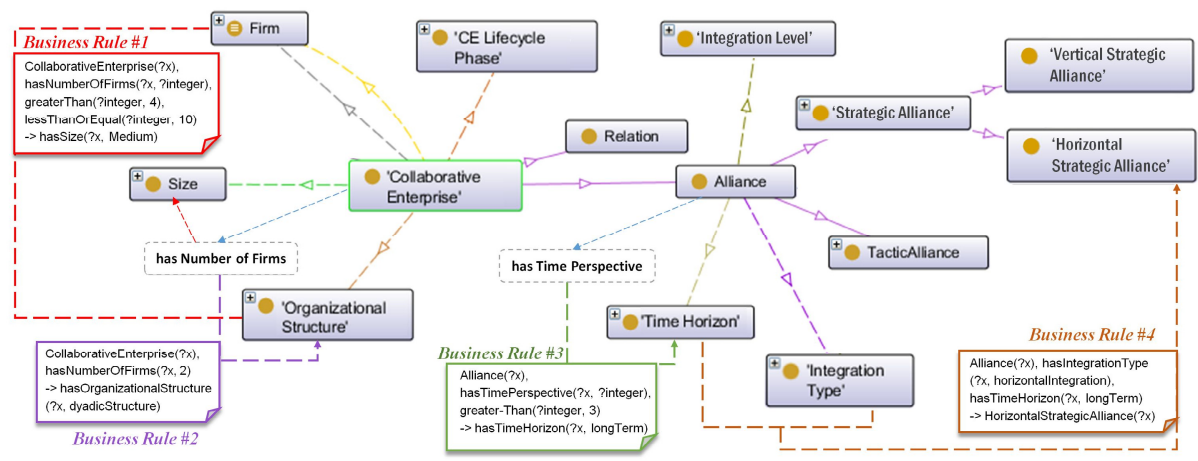

Fig. 2. Representation of the business rules

Moreover, the alliance type is defined by means of a business rule that takes into account the integration type and the time horizon. The last one is useful in order to distinguish between strategic alliances (medium and long term) and tactic alliances (short term), while the integration type helps in order to divide horizontal alliances from vertical ones. In more detail, with the rule "Alliance(?x), hasIntegrationType(?x, horizontalIntegration), hastimeHorizon(?x, longTerm) -> HorizontalstrategicAlliance(?x)" we assert that if an alliance has a horizontal integration as well as a long-term time horizon, then it can be considered a horizontal strategic alliance. Also, the classification based on the organizationalstructure is based on the number of firms, the participation of firms to the $\mathrm{CE}$, the existence of a focal firm and the presence of other forms of organizational structure. In more detail, the following rule "CollaborativeEnterprise(?x), hasNumberoffirms (?x, 
dyadicStructure)" asserts that if a CE is composed by exactly two firms, then it has a dyadic structure. Moreover, the rule "CollaborativeEnterprise(?x), hasfocalfirm(?x, ?y), haspart(?x, ?y), hasNumberoffirms(?x, ?integer), greaterThan(?integer, 2) $->$ hasorganizationalstructure(?x, organizationWithFocalFirm)" asserts that if CE is composed by more than two firms, has a focal firm and this focal firm is part of the CE, then the $\mathrm{CE}$ has an organizationalstructureWithFocalfirm. Finally, the rule "CollaborativeEnterprise(?x), hasNumberoffirms(?x, ?integer), greaterThan(?integer, 2), DifferentFrom (?y, organizationWithFocalfirm) -> hasorganizationalstructure(?x, organizationWithoutFocalFirm)" asserts that if a CE is composed by more than two firms and it can't be associated with the class organizationalstructureWithFocalFirm, then it has an organizationalstructureWithoutFocalFirm. The clause DifferentFrom was used in association with an owl : allDifferents axiom declaration to assess that the individual organizationalstructureWithFocalFirm is different from organizationalstructureWithoutFocalFirm. That clause was chosen because SWRL does not support negation as a failure, being a monotonic language.

\subsection{Validation}

In order to assess the quality of the proposed ontology, a way to evaluate how accurate is the encoded knowledge is needed. As a consequence, competency questions (CQs) were introduced in Section 3 in order to model our knowledge base accordingly. The ontology should contain enough information to be able to answer to CQs and these aspects have to be checked during validation. The importance of CQs during the entire ontology development and validation process was highlighted by Presutti et al. in [26]. According to them, CQs must be formalized into queries that have to be run against the ontology, so that query outcomes must be compared with the expected results. However, translating CQs into formal queries is not a straightforward task [27], since many aspects should be taken into account: presence of rules, necessity of considering inferred knowledge, etc. Some alternatives are available and the user should select the most suitable one according to its application scenario. SPARQL (Simple Protocol and RDF Query Language) [28] has high expressivity and allows to query and manipulate any RDF graph but considering inferences can be troublesome (unless SPARQL 1.1 entailment regimes are used). DL Queries [29] are more suitable to OWL class expressions and allow to capture inferences but cannot manage SWRL rules. SPARQL-DL [30] combines the best features of SPARQL and DL Queries but it is not a W3C standard and its future is still not very clear. Finally, SQWRL [31] is a SWRL-based language capable of querying OWL ontologies thanks to an ad hoc library of SWRL built-ins. Therefore, we used SPARQL to check SCQs and SQWRL to ascertain that CCQs can be answered as well (see Section 3). For the sake of brevity, an example from each of the two approaches is reported. The SCQ.2 was associated with the features of a $\mathrm{CE}$ : it can be translated into the following SPARQL query: 


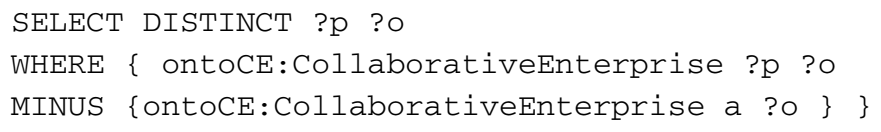
ject domain. In the same way, CCQ.3 aimed at classifying a CE having a time perspective of more than 3 years and a vertical integration. It can be rendered in SQWRL as in the following:

CollaborativeEnterprise(?x),

hasIntegrationType(?x, verticallintegration), hastimeHorizon(?x, ?integer), greaterThan(?integer, 3)

$->$ sqwrl: select (?x)

Where all the CEs satisfying the conditions mentioned above are enlisted.

By adopting this methodology, we managed to verify that all the CQs can be answered by selecting a proper querying approach

\section{Discussion and Conclusions}

In this work, we introduce a semantic model for a large class of Collaborative Networks (CNs) defined as Collaborative Enterprises (CEs), in order to enable their understanding and analysis. The emphasis of the paper is on structuring the characteristics of CEs with respect to size, integration type and level, time horizon and so on. The classification of the instances is achieved by means of business rules. The development of the Collaborative Enterprise ontology, supported with business rules, enable the semi-automatic classification of CEs, which, if associated with a KPI Ontology brings to the development of an interpretative framework able to support both CEs and firm managers to understand which KPIs to use and how to integrate them in a performance framework.

Future works will include the online publication of the ontology in order to make the collection generally accessible to the community of researchers and practitioners interested in performance indicator modeling. For the purpose of our research, the ontology will be completed with a set of suitable software components with the aim of supporting users to effectively build, analyze and manage CEs.

\section{References}

1. FInES Research Roadmap Task Force: Future Internet Enterprise Systems (FInES)Research Roadmap (2010)

2. Bititci, U.S., et al.: Performance Measurement: Challenges for Tomorrow. Int. J. Manag. Rev. 14, 305-327 (2012)

3. Bititci, U.S., et al.: Creating and managing value in collaborative networks. Int. J. Phys. Distrib. Logist. Manag. 34, 251-268 (2004)

4. Kaplan, R.S., et al.: Managing Alliances with the Balanced Scorecard. Harv. Bus. Rev., 114-121 (2010)

5. Bititci, U.S., et al.: Collaboration: a key competence for competing in the 21 st century (2008) 
6. Parung, J., Bititci, U.S.: A conceptual metric for managing collaborative networks. J. Model. Manag. 1, 116-136 (2006)

7. Popova, V., Sharpanskykh, A.: Modeling organizational performance indicators. Inf. Syst. 35, 505-527 (2010)

8. Guarino, N.: Understanding, Building and Using Ontologies: a Commentary to Using Explicit Ontologies in KBS Development. Int. J. Hum. Comput. Stud. 46, 293-310 (1997)

9. FInES Task Force on Collaborative Networks and SOCOLNET: Taxonomy of Collaborative Networks Forms (2012)

10. Bertolazzi, P., et al.: An approach to the definition of a core enterprise ontology: CEO. OES-SEO 2001. In: International Workshop on Open Enterprise Solutions: Systems, Experiences, and Organizations, pp. 14-15. Citeseer (2001)

11. Plisson, J., et al.: An ontology for virtual organization breeding environments. IEEE Trans. Syst. Man, Cybern. Part C Appl. Rev. 37, 1327-1341 (2007)

12. Ermilova, E., Afsarmanesh, H.: Modeling and management of profiles and competencies in VBEs. J. Intell. Manuf. 18, 561-586 (2007)

13. Madni, A.M., et al.: IDEONTM: An extensible ontology for designing, integrating, and managing collaborative distributed enterprises. Syst. Eng. 4, 35-48 (2001)

14. Ye, Y., et al.: An ontology-based architecture for implementing semantic integration of supply chain management. Int. J. Comput. Integr. Manuf. 21, 1-18 (2008)

15. Livieri, B., Bochicchio, M.A.: Information Systems and Performance Management for Collaborative Enterprises: a proposal. In: 26th CAiSE FORUM, pp. 1-8 (2014)

16. Livieri, B., Bochicchio, M.A.: Performance Modeling for Collaborative Enterprises: Review and Discussion. BIR (2014)

17. Dietz, J.: Enterprise Ontology: Theory and Methodology. Springer (2006)

18. Hitzler, P., Parsia, B., Rudolph, S. (eds.): OWL 2 Web Ontology Language Primer, 2nd edn. (2012)

19. Van Heijst, G., Schreiber, A.T., Wielinga, B.J.: Using Explicit Ontologies for KBS Development. Int. J. Hum. Comput. Stud. 46, 183-292 (1997)

20. Breuker, J., et al.: Developing Content for LKIF: Ontologies and Frameworks for Legal Reasoning. In: JURIX 2006 19th Annu. Conf., vol. 152 (2006)

21. Pedrinaci, C., Domingue, J., Alves de Medeiros, A.K.: A Core Ontology for Business Process Analysis. In: Bechhofer, S., Hauswirth, M., Hoffmann, J., Koubarakis, M. (eds.) ESWC 2008. LNCS, vol. 5021, pp. 49-64. Springer, Heidelberg (2008)

22. Protégé Ontology Editor, http://protege. stanford. edu

23. Sirin, E., et al.: Pellet: A Practical OWL-DL Reasoner. Web Semant. Sci. Serv. Agents World Wide Web. Softw. Eng. Semant. Web. 5, 51-53 (2007)

24. Eiter, T., Ianni, G., Polleres, A., Schindlauer, R., Tompits, H.: Reasoning with Rules and Ontologies. In: Barahona, P., Bry, F., Franconi, E., Henze, N., Sattler, U. (eds.) Reasoning Web 2006. LNCS, vol. 4126, pp. 93-127. Springer, Heidelberg (2006)

25. Horrocks, I., et al.: SWRL: A semantic web rule language combining OWL and RuleML. W3C Member Submission 21, 79 (2004)

26. Presutti, V., Daga, E., Gangemi, A., Blomqvist, E.: eXtreme design with content ontology design patterns. In: Proc. Workshop on Ontology Patterns, Washington, DC, USA (2009)

27. Damljanovic, D., Agatonovic, M., Cunningham, H.: Natural language interfaces to ontologies: Combining syntactic analysis and ontology-based lookup through the user interaction. In: Aroyo, L., Antoniou, G., Hyvönen, E., ten Teije, A., Stuckenschmidt, H., Cabral, L., Tudorache, T. (eds.) ESWC 2010, Part I. LNCS, vol. 6088, pp. 106-120. Springer, Heidelberg (2010)

28. SPARQL 1.1 Query Language. W3C Recomm., http://www.w3.org/TR/ sparql11-query/

29. DL Query 1.1.0, http: / /protegewiki.stanford.edu/wiki/DL_Query_1.1.0

30. Sirin, E., Parsia, B.: SPARQL-DL: SPARQL Query for OWL-DL. OWLED (2007)

31. O'Connor, M.J., Das, A.K.: SQWRL: A Query Language for OWL. OWLED (2009) 